\title{
Achieving impact in ergonomic research
}

\author{
Nick McDonald ${ }^{\mathrm{a},}{ }^{*}$, Marie Ward ${ }^{\mathrm{a}}$ and Rabea Morrison ${ }^{\mathrm{a}}$ \\ ${ }^{a}$ Aerospace Psychology Research Group, Trinity College Dublin, College Green, Dublin, Ireland
}

\begin{abstract}
People are central to system functioning and this role has been dramatically extended by new information technology. This makes possible the fundamental transformation of processes across systems of systems. Can Ergonomics research play an effective role in systems integration innovation? To have real world impact on such problems the system of R and D needs to sustain strong designs that address the functionality of socio-technical systems and support the implementation of innovations, taking into account the complexity of change, the importance of values of dignity and trust, and creating a common understanding amongst all stakeholders to enable design for operations. Strong research designs are more expensive, difficult, risky and prolonged than more commonly practiced weaker designs. They require active engagement with the industrial or service provider and involve overcoming cultural and other barriers to effective implementation and change. To create a virtuous cycle of research-generated impact it is necessary for strong designs to be well supported in the research community and for real world impact to be central to performance criteria of research excellence. Unfortunately neither of these criteria appear to be fulfilled. To ameliorate this, deficiencies in the full cycle of systems integration innovation need to be addressed.
\end{abstract}

Keywords: research impact, systems integration, implementation, design for operations, trust

\section{Introduction}

People are central to system functioning; they have an important, often taken for granted, role coordinating processes, as well as task-related roles. Information technology has dramatically extended the reach of this co-ordination role, enabling the transformation, not just of an organization, but of systems-of-systems - for example the many components that make up the aviation system. Complex system interactions are implicated when things go wrong. To cope with this complexity Ergonomics and Human Factors have to transform their theory and practice to ensure relevance to these problems and generate the leverage to change the situation. Ergonomics, as a human or social science, has as its subject matter the organized goal-directed activity of people in the world. The need to broaden the focus of the discipline to organized human activity across quite wide ranging and complex systems highlights the problems of building a science that can not only interpret that reality, but also understand its underly- ing causal dynamics to the extent of enabling some prognosis of future outcomes (including resilience to meet future challenges) and creating an informed basis for design and intervention in constructing the socio-technical systems of the future.

However, it is not enough to have a good theory about socio-technical systems; the conditions for implementation in the relevant industrial or services environment have also to be favorable for human systems design, for better supply, planning and management and for improvement, integration and organizational change. Those responsible for the design and management of the system are accountable for the system outcome - that it is better, safer, cheaper, higher quality or environmentally friendly as the case may be. This in turn means that we have to understand not just a linked series of local processes but how that linked set of activities across a wide organizational space delivers (or not) the system outcomes that are desired. This concerns all those organizational processes that plan, deliver and manage all the people-related functions, together with the collective

${ }^{*}$ Corresponding author. E-mail: nick.mcdonald@tcd.ie, phone: +35318961471 
meanings and values that inform people's understanding and action. Lewin's adage - 'there is nothing so practical as a good theory' needs to be pushed to its limit - the process of implementation needs to be organized into a practical theory of change.

A third precondition for impact concerns the research and development system itself - the goals of research for the different stakeholders (researchers, their institutes, the commissioners of research and its clients, journals and publishers), together with the opportunities and constraints that shape the particular methodologies and theoretical frameworks deployed, which ultimately determine the outcome of the research. This is what defines the possibility of impact, from the supply side, and feeds the transfer of knowledge or technology into the creation of a new product or process. This is research-driven innovation.

These three aspects are represented in figure 1 as a virtuous cycle of mutual interaction. Scientific practice, through its theory and methods, defines the impact or change that needs to be achieved. Achieving that impact on industry or services requires knowing how to do it and what to overcome. The deployment of powerful scientific methodologies needs to be supported and valued by the research and development system and this needs to be reinforced by accountability for, or recognition of achieving impact. How well does this work in practice? This will be considered under five headings: core theory and methods about system functioning; implementation; the role of culture; design for operations; and the innovation process.

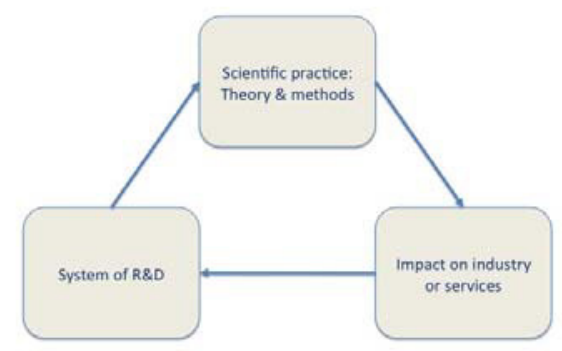

Figure 1: A simple model of research-based innovation

\section{Mutual interaction of theory and methods, impact and system of $R \& D$}

\subsection{Theory and methods}

Understanding the functionality of how a system works is the key to managing that system more effectively, and to comprehending how it is possible to change the system to achieve better outcomes, or how to design a future system to operate in a way that transcends current practice. Understanding and managing the complexities and risks of operational systems, being able to achieve change and to design future systems are the core capabilities to meet the challenges outlined above. Paradoxically, while we spend our lives in organizations, constrained by what they permit us to do, while fulfilling, to a greater or lesser extent, those organizations' purposes, we lack a good theory of the functionality of organizations. Thus a rich account of the human role in productive processes; is lacking in most organizational theory, is beyond the more local focus of most Ergonomic theory, is too deterministic for cultural theorists, and too human-oriented for business process engineers.

An account can be given of functionality at different levels of explanation:

- What causal factors can explain the outcome?;

- Can the outcome be predicted?;

- Can the factors that influence the outcome be changed?;

- Can change be managed so that targeted outcomes can be attained?;

- Can the change that has been achieved be evaluated (i.e validate the model)?

It is not necessary to assume that systems are totally determined or that we can comprehend all the determinant influences on them. The point is to push the boundaries of what can be explained within a causal framework, so that we can better understand the degree of influence of those factors whose parameters are not well understood and are therefore not properly within the explanatory model. These include both unpredictable external influences on the system as well as internal 'emergent' factors.

Understanding cause requires a strong methodology that optimally includes the following four elements. The first is a methodology for analysing the functional and causal relationships within the system. The logic of such a functional account is to describe a mechanism, or set of mechanisms that can provide something approaching necessary and sufficient conditions for an event, or movement in the system to 
occur. The second concerns the study or analysis of real operational data that provides information on what people are doing and what the system (as a whole or in parts) is doing. This provides the basis for causal influence of 'constant conjunction' or correlation in the movement or variation of different elements. Because the proliferation of potential types and sources of data in organizations is vast, it is necessary to fulfill the first condition in order to make sense of the second; without some explanatory framework it is impossible to know what to look for, how to combine data, or how to interpret patterns in the data. The third requirement concerns the triangulation of different methods and measures; because socio-technical systems are multidimensional and because data rarely directly represents the dimensions of theoretical interest and may be biased and contaminated, it is important to be able to compare and interpolate between different data sources. The final requirement of a strong methodology is a longitudinal design that permits the measurement of change over time.

Relatively weak designs or methodologies are either unable to address the dynamics of the movement of systems over time in a definitive way, or they focus on part of the system but not its central causal mechanisms. In retrospective or cross-sectional designs robust causal inference is difficult or impossible. These include investigations, post-hoc case studies and comparative surveys in one time frame. Other studies are epiphenomenal in that they focus on a non-determinant aspect of the system that may be more a reflection of than a direct representation of the system such as culture, climate, attitudes or other individual or collective cognitions. Others are local and focus on the task or the technology interface rather than overall system relations. Other approaches are techno-centric in that they focus on the relationships between the technology elements in the sociotechnical system, but neglect the social relations that complement these.

Strong designs are inherently more difficult: they are more complex, they are more resource intensive, they take a longer time and they require active engagement with the system being studied. This normally means the active participation of the organizations and institutions that 'own' or control the system in question.

\subsection{Implementation}

The literature on organizational change demonstrates that, against different criteria and outcomes, only a minority of major change initiatives (typically between 30 and 50 percent) have a positive outcome $[11,33,17,30,22]$. From some of the few longitudinal studies of change, Pettigrew shows how change is complex, frequently opportunistic, and depends on the balance of capacity within the organization with the opportunity that the organization's environment brings [31-32]. In the aviation system, a series of European projects [e.g.13] have analysed the difficulties that organizations have in achieving effective change even in response to serious safety incidents.

The most simplistic model of change is one of action and reaction, stimulus and response. Change involves initiating or proposing an action, and this in turn evokes reaction, which can be acceptance (or at least acquiescence) or rejection. A new pattern of activity is consolidated: the new activity can be reinforced, increasing its likely repetition; or it can evoke a negative reaction blocking progress and consolidating the status quo. However, what can appear as resistance to change may be more complex than it seems. According to Dent and Goldberg [10], often individuals are not really resisting the change itself, but they may be resisting the reduction in status, pay, or loss of comfort they perceive as being associated with it. They argue that, "it is time that we dispense with the phrase resistance to change and find a more useful and appropriate model for describing what the phrase has come to mean - employees are not wholeheartedly embracing a change that management wants to implement" (p. 26). Strebel [39] suggests that management should view how change looks from the employees' perspective; and Kegan and Lahey [16] argue that change itself is not challenged, but rather is it resisted, or not implemented at all because the employee faces additional issues or concerns related to the change. When an employee's hidden competing commitment is uncovered, "behavior that seems irrational and ineffective suddenly becomes stunningly sensible and masterful - but unfortunately, on behalf of a goal that conflicts with what you and even the employee are trying to achieve" (p. $85)$. One lesson from this is that different people hold different stakes in the process and outcomes of change.

A more complex model of change involves a benign spiral of growth in which a new understanding 
complements new opportunities to act in a different way, such actions transforming, while also reproducing, the social system. As this new pattern of interaction becomes normative it is embedded in an evolving culture, being reflected in commonly held meanings and values. This is the pattern of the learning organization, popularized by Senge [37]. Unfortunately it is not easy to generate this virtuous cycle. More common are 'cycles of stability' [23] in which a lot of organizational effort goes into addressing problems and difficulties (for example, quality or safety incidents) but there is little fundamental change to address the core issues of the problem. Existing relationships and interactions are reinforced, established understanding is confirmed, cultures and subcultures are strengthened. Ward et al [46] provide an example of overcoming these cycles of stability to generate effective change.

In the most radical version of change, the accumulation of many specific local changes can ultimately transform the fundamental relationships of the system, creating new patterns of interaction, transformed understanding, new problems and issues to confront and changed culture. This is the pattern of revolutionary change, well exemplified by Kuhn's seminal work, 'The Structure of Scientific Revolutions' [19]. Sometimes new technologies create the potential to stimulate and enable such a transformative change in organizations or the way in which operational systems work. It is tempting to think that technologies determine the way they are used and that the way they are used determines the mode of social interaction for that production system. However this is too simplistic - technologies offer different opportunities and constraints; some challenges in the design of effective socio-technical systems to exploit this potential are considered in section 2.4.

Change is complex and multidimensional and successful implementation should not be taken for granted. A framework for planning and managing change should start with the goals and strategy of the implementing organizations, together with the process structure and support systems that can deliver the required outcome. The use of operational data to understand how the system is performing now and where it needs to go provides a necessary link between strategy and operational change. A major barrier is often the capacity to function as a 'joined-up' system, transcending functional 'silos' This needs to be supported by appropriate competence in depth and across the organizations involved. Often peoplemanagement skills are relatively undeveloped but the complementary resources of both internal and exter- nal change agents can help create a critical dynamic of change [20]. Thus the process of change should be considered a core part of the equation of implementation.

\subsection{The role of culture}

Culture is a critical force for stability in social systems. Therefore, its roles need to be understood if change is to be managed effectively. For example: the exploration of professional culture can reveal hidden ways in which people compensate for system inadequacies, keeping the system going using informal norms which sometimes contradict the formal rules. When things go wrong such informal practices are often hidden from official scrutiny - thus distorting the potential to find out what really happened and how to change the system to prevent future failures. It is useful to think of culture as comprising, at least in part, a collective reflection of 'how things work' (rather than just of 'how things are done around here'). From this perspective it provides a rationale for why we act in a certain way, as well as how others interact with us. In terms of the cultural acceptability of change, this draws attention to the importance of delivering a more effective system in the resolution of change - 'does it work better? Does it work better for me?' It is relatively easy to promise a vision of change, but more difficult to deliver tangible benefits to those who participate in the change. Delivering release from frustration, by solving recurrent problems that interfere with the smooth flow of work, was a critical success factor in the change program described by Ward et al [46].

Culture is also about values. Values are transcendental in that they reflect an enduring quality that we attribute to situations, objects or relationships. There are commonly two discourses about values: one treating the value as an absolute quality, and end in itself; and the other seeing values as instrumental in achieving broader social goals. Two key issues concern the value of dignity in social relations and how trust is embedded in the way the social system works.

Bolton [7] argues that dignity is

“...overwhelmingly presented as meaning people are worth something as human beings, that it is something that should be respected and not taken advantage of and that the maintenance of human dignity is a core contributor to a stable "moral order"” p.7) [7]. 
She goes on to stress however that dignity at work is problematic - when we sell our labor do we also relinquish autonomy, freedom, equality and often our well-being - the very ingredients of life that have been most commonly associated with human dignity.

The issues of dignity at work has not been explored from a psychological or an ergonomic viewpoint. It has unfortunately been associated in some parts of the world with anti-bullying but is fundamentally much greater than this. There have been texts written on the subject from a sociological position [14] and from a business perspective [7]. We need however to look at dignity, particularly in relation to trust, safety and system change.

When workers treat each other with respect and dignity then they can develop trust in each other [13]. Sitkin and Stickel [38] note that sociologists and psychologists have proposed that trust is an element that makes work in organizations possible [5] through its effects on cooperation [e.g.18], interpersonal and group solidarity [e.g. 4], and facilitating social infrastructure [49]. The positive contribution of trust in relation to safety has also received a lot of attention recently $[8,35,48,44]$.

Flin and Burns [12] and Conchie and Donald [9] have shown that safety-specific trust appears to develop from three trustworthiness factors relating to ability, integrity and benevolence. Tan and Chee [40] and Conchie and Donald [9] however suggest that integrity and benevolence are the most important determinants of safety-specific trust. Integrity reflects the extent to which the person is honest and open in sharing information and benevolence reflects the extent to which the person shows concern for another's welfare [21]. Both of these concepts could be said to relate to the concept of dignity.

Dignity and trust in relations within organizations appear to play a critical role in enabling change and improvement to happen. Following a meta-analysis of three large scale ergonomics / human factor interventions in aircraft maintenance organizations Ward [45] found that organizational change was not possible when people were not being treated with dignity in the first instance. She found that resistance behaviors were not objections to change per se but to how people were being treated at the time and were what Hosking [15] terms 'the local rational response'. Ward [45] demonstrated the importance of understanding the systemic basis of trust in change initiatives - in particular the existence of common goals, open communication, clearly understood ways of working (formal rules or procedures and normal ways of working being not too different) and having an opportunity for feedback and review. A history of mistrust also carries forward into current relationships. Trust also involves mutuality, so it is important to consider how change delivers benefit to all who have a stake in it.

If the interests of different stakeholders are to be properly considered then trust and dignity are irreducible values. But this argument is not a simple utilitarian one - that you always have to treat people with dignity in order to get effective change. However, in so far as social cohesion is often important in addressing difficult challenges, so trust and dignity have to be part of the utilitarian equation as well as values in their own right. This applies to all stakeholders: employees, suppliers, customers, team partners, amongst others.

\subsection{Design for operability}

Manufacturers, in moving from selling technology to providing services through technology, have to learn to 'design for operations' and anticipate the functional impact of their technologies. For example, in the aviation system, operability as a new concept integrates business drivers across different domains of the aviation lifecycle and aims to design the lifecycle around the delivery of an optimized operation of an airline fleet. For example, in the aviation community Airbus defines operability as "an aggregate measure of the aircraft's availability, operational reliability and maintenance related costs" [43] In contrast to attributing these metrics to specific departments or domains in or across aviation organizations, such as maintenance, flight operations, engineering support, operability is put forward as a system measure that emphasises the interrelatedness of the system processes. However to implement this innovation necessitates a full understanding of the world of operations. This requires not only an effective collaboration of different stakeholders across the lifecycle (design, operations, maintenance), but also a strong framework which systematically captures, integrates and deploys this knowledge in system engineering to achieve the desired outcomes or impact.

\subsubsection{Design standards}

"New systems and new technologies can dictate a step change in the operation. They do not just change the jobs that people do - they can transform the whole process. It is this process transformation which delivers the step change in operability. Therefore the manufacturer has not only to engage with the 
Human-Machine-Interface, but also with how the technology fits into and facilitates the whole operational system.'[25] Thus, the operation has to be systematically represented in any design approach and standard.

As an illustration of this, the work in the TATEM project can be cited [e.g. 41, 42]. The project as a whole aimed at reducing the maintenance element of the direct operating cost of an airline by introducing innovative health monitoring and management technologies that increase the control over the maintenance schedule, at fleet level as well as in a specific maintenance situation/process. The TATEM systems architecture is based on the Open systems architecture condition based maintenance (OSA CBM) standard [26]. The OSA CBM standard is designed as a hierarchical structure that drives information management from data acquisition to presentation. However, this standard stops at the presentation layer - it does not cover the use of this information nor the way in which such new information can transform the basic dependencies which govern the processes of maintenance. Hence it was proposed to add an operational activity layer [e.g. 27] to transparently link the core technology development into the operational system within which it is meant to deliver to metrics such as operability. It is in this layer, through the power of new information systems to transform processes, that the greatest leverage to improve operability is found.

In order to implement each layer the knowledge of different groups of stakeholders in different places and belonging to different organisations is required. Morrison et al. [27] discuss the significant task of creating a shared understanding of how the technology impacts their respective interdependent processes.

\subsubsection{Bridging the knowledge gap}

Designing for operations requires an understanding of how the operational system actually works which is only partially represented in formal descriptions available to designers (e.g. SOPs, process manuals). Designers rarely have operational experience, and the design process is usually remote (geographically, commercially, organizationally) from the operational situation.

Bridging this gap between design and operations cannot be managed by representing the operational sector solely as end-users. The operational sector is a stakeholder in the design process which needs to be systematically represented in each step of any adopted system engineering approach, e.g. the tradi- tional $\mathrm{V}$ cycle runs from initial concept to requirements, prototype to development, and then back through levels of demonstration, validation and evaluation. Delivering operability is a joint effort and shared responsibility between partners.

To understand how a system actually works, one cannot only rely on explicit knowledge but one also needs access to knowledge that is tacit, i.e. not formalized and explicit and not easily accessible. Nonaka [e.g. 28, 29,] has put forward a framework for accessing such knowledge and deploying it in product innovation. The Human Factors framework developed in a series of collaborative system engineering projects [e.g. 41, 42] draws on Nonaka's work and adapts its principles to facilitate a transformation of the initially separate understanding of each stakeholder into an integrated vision of the potential impact of the new technology [27, 41-42]. The core principles of this approach are:

- The capacity to model the operational system in a naturalistic and ecologically valid manner, capturing the 'tacit knowledge' of the operational community.

- The ability to transfer and transform this knowledge into a capability to envisage and design how a future system might operate.

This knowledge transformation process involves getting the key stakeholders from design and operations together in an active engagement collaboratively developing a new concept.

\subsubsection{Role of networks}

Knowledge services support the interchange of process knowledge between stakeholders, learning from each others' experience and sharing operational data. To continuously drive innovation, a knowledge network needs to be set up that proactively transforms knowledge to meet not only current but also emerging challenges. Such knowledge networks can operate in different ways, including clusters of excellence, training and education and research.

\subsubsection{Design process}

The design process requires a strong operational model from the beginning to enable it to effectively deliver system metrics such as operability.

"It is important to understand how new technologies can transform the functioning of newly integrated operational systems in order to deliver these outcomes in a coordinated and effective manner. To do this, we require new capabilities to model operational systems in a rich and comprehensive way and 
to gather and integrate a range of operational data sources to enable in-depth systemic analyses. Successful innovation depends upon transforming this operational data into useful knowledge and transferring it to where it can influence both new technologies and new operational systems. Thus managing knowledge across the system lifecycle becomes a central enabler of system development." [24]. In a system like aviation where design and operation are quite remote from each other in terms of both geography and professional culture, this is a formidable challenge.

\subsection{Innovation}

It is well known that the relationship between firms and between firms and research and development organizations is a key factor enabling innovation. Both clusters of organizations [34] and communities of practice $[36,47]$ are, in different ways, important in the dissemination and uptake of new knowledge. While much of the research into systems of innovation focuses on product innovation, process innovation (new ways of doing things) is also important. Indeed Ergonomics and Human Factors are, arguably, the key link between product and process innovation - in so far as the usability of a product is related to the use of that product in a process to produce another product or service. Thus the usability of a tool is translated into its use value in a task and this in turn is translated into the operability of the technology in producing an effective process outcome. Because the subject-matter of Ergonomic research concerns the functioning of real socio-technical systems, the way in which such research has impact and contribute to innovation needs careful consideration. Best [6] describes a number of models of the innovation process. The classic model is labeled 'Laboratory-push' innovation and is typical of big business organizations (see figure 2). In this model, it is the job of science to do basic research in laboratories; new product ideas from this research will be taken up by design engineers in a developmental program of increasingly focused application to lead eventually to a product.

An alternative model, systems integration innovation (figure 3) seems much more appropriate for understanding how Ergonomics can have impact, particularly when considering the system role of new technologies. This model starts with a product or component (it could equally well be a process); building a complex product leads to problems of integra- tion and such problems can lead to basic research questions which need to be resolved for integration to succeed. In terms of the role of research it is almost the opposite of the laboratory-push model. Research needs to be actively and intimately involved in the innovation process whether this revolves around a new product, a new process, or both.

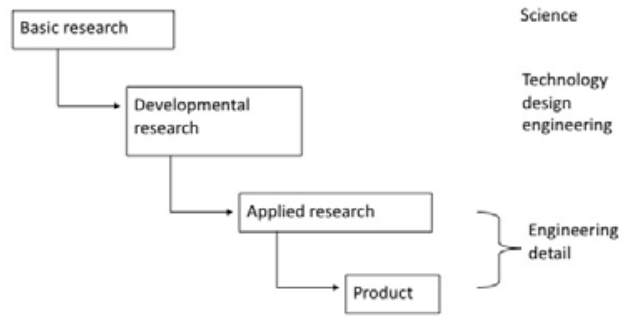

Figure 2: Laboratory-push innovation (taken from Best [6])

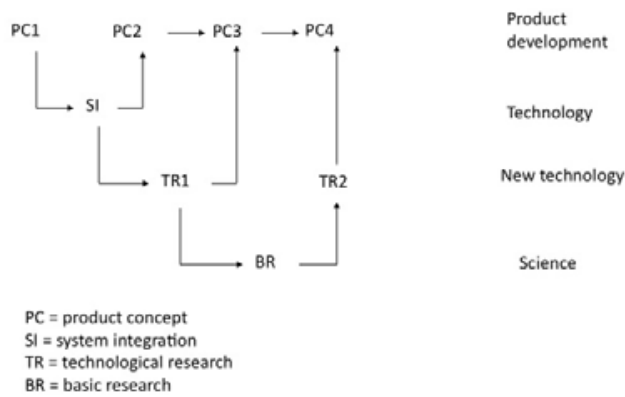

Figure 3: Systems integration innovation (taken from Best [6])

This model favors close and long-term links between research and industry partners in an innovation cluster that fosters mutual understanding and the identification of problems and potential novel solutions.

Such links have a better chance of sustaining the strong designs that are the hallmark of a more powerful theory, but there are also difficulties. The research capability may be unevenly distributed across the cluster: for example, unlike most large manufacturing organizations, operational and service delivery or- 
ganizations do not tend have their own research and development departments. This means that independent research organizations or universities have to play this role. Research often has to transcend organizational boundaries.

A research process based on strong designs that can deliver impact is difficult: it is resource intensive, because of the complexity of real world sociotechnical problems; it is relatively risky, because its progress depends on external constraints that can severely delay or derail the research; it is often opportunistic to cope with this constraint but this can lead to a more 'messy' research process with less clear outcomes; and with a longitudinal trajectory, such research requires an extended time commitment. Furthermore, the research process itself requires investment in those factors that are critical for effective implementation and change and necessitates attention to be paid to cultural values like dignity and trust that are important in achieving effective outcomes.

Unfortunately these characteristics of real world research are in potential conflict with dominant performance criteria that are used to manage the research process. These criteria prioritize frequency of publication in 'high impact' peer review journals and measure impact through citations. Pressure for frequency of publication militates against using strong designs as defined above, because they are more expensive, difficult and risky and take longer than weak designs. Within the system integration model of innovation, the primary and most influential channel of impact is probably not through journal publication. Rather it works through the direct application of research-based concepts and methods in real systems, which are then disseminated by codes of practice, communities of practice and other industrial networks. The failure to implement the proposed Australian Research Quality Framework for measuring research impact illustrates the real difficulty in reconciling measures of research impact in the real world with bibliographic methods. Thus it seems that current publication and citation policies encourage research with relatively low impact in the real world. An anecdotal example of the mismatch of expectation between research and industry concerns the recent strongly expressed view of the R\&D department of a major manufacturer concerning its long-term university engineering school partner - the latter's horizons are too short term, caught up in this and next year's publications, so that it is difficult to get it engaged in industry's strategic research agenda.

\section{Conclusions and recommendations for the future}

The argument has been that strong research designs in Ergonomics are necessary to achieve impact but that these are more expensive, difficult, risky and prolonged than common weaker designs. Strong research designs require active engagement with the industrial or service provider host of the process concerned. This in turn requires attention be given to complex multidimensional implementation processes and overcoming cultural and other barriers to effective implementation and change. To create a virtuous cycle of research-generated impact, as illustrated in figure 1, it is necessary for strong designs to be well supported in the research community and for real world impact to be central to performance criteria of research excellence. Unfortunately neither of these criteria appear to be fulfilled. Thus relatively weak designs and low real world impact appear to be the norm.

Improving the current innovation model for Ergonomics research demands attention to the following aspects:

- Goals and objectives between the academic or research side and industry need to be better aligned. The main performance management framework governing research may well be undermining real world impact, particularly where such research requires active engagement with real social systems.

- The research process itself needs to be reinforced with a stronger methodological base built around a core functional concept of sociotechnical systems, complementing the current predominant focus on cognitive or cultural analysis.

- Ergonomic or Human Factors competence in industry needs to be enhanced to support the difficult tasks of implementation and change. Researchers also need better understanding and experience of the operational side of industry.

In so far as there is a cultural gulf between research, product design, and operations and services, if there is an imperfect understanding of each others' roles and ways of working, a misalignment of performance goals, few really effective channels of communication across these silos, and a lack of feedback about effectiveness, these are the conditions that make for a greater potential for mistrust, according the Ward's analysis of organizational barriers to change [45]. A potentially virtuous cycle of innova- 
tion is in danger of becoming a cycle of stability with relatively low impact unless Ergonomics research can improve its leverage over large-scale challenges of system transformation.

\section{Acknowledgements}

Research reported in this article has been supported by the European Commission framework program.

\section{References}

[1] Adams (1994 - 1999) Human Factors in Aircraft Dispatch and Maintenance. Under the EU BRITE-EURAM Programme. Contract No. BRPR-CT95-0038, Project No: BE95-1732. Brussels: European Commission.

[2] Adams2 (2001 - 2004) Human Factors in Aircraft Dispatch and Maintenance. Under the EU BRITE-EURAM III Programme for Industrial Materials and Technology. Project No: GRD1-2000-25751. Brussels: European Commission.

[3] AMPOS (1999 - 2001) Aircraft Maintenance Procedure Optimisation System. Under the EU ESPRIT Programme. Project No: 29053. Brussels: European Commission.

[4] Barber, B. (1983). The logic and limits of trust. New Brunswick, NJ: Rutgers University Press.

[5] Barnard, C.I. (1938). The functions of the executive. Cambridge, MA: Harvard University Press

[6] Best, M.H. (2001) The New Competitive Advantage: The Renewal of American Industry. New York: Oxford University Press.

[7] Bolton, S. (Ed) (2007) Dimensions of Dignity at Work. London: Elsevier.

[8] Conchie, S.M., Donald, I.J. \& Taylor, P.J. (2006) Trust: missing piece(s) in the safety puzzle. Risk Analysis 26, 10971104.

[9] Conchie S.M. and Donald, I.J. (2008) The functions and development of safety-specific trust and distrust. Safety Science 46, 92-103

[10]Dent, E. B. and Goldberg, S. G. (1999). Challenging 'resistance to change,' Journal of Applied Behavioral Science, March, Vol. 35, No. 1, 25-41

[11]Dent and Powley (2001). Employees Actually Embrace Change: The Chimera of Resistance. Journal of Applied Management and Entrepreneurship, September 26, 2001

[12]Flin, R. and Burns, C. (2004) The role of trust in safety management. Human Factors and Aerospace Safety 4, 277287.

[13] Gratton, L. (2003). The Democratic Enterprise. Liberating your business with freedom, flexibility and commitment. London: Prentice Hall.

[14]Hodson, R. (2001). Dignity at work. Cambridge: The Press Syndicate of the University of Cambridge.

[15] Hosking, D.M. (2004) Change Works: A critical construction. In J. Boonstra (Ed) Dynamics of Organizational Change and Learning. Chichester: Wiley.

[16] Kegan, R. and Lahey, L. (2001, Nov). The real reason people won't change. Harvard Business Review 85-92

[17] Kotter, J. P. (1995, Mar-Apr). Leading change: Why transformation efforts fail. Harvard Business Review, Vol. 73, No. 2,
March/April, pps. 59-67.

[18] Kramer, R.M. (1993) Cooperation and organizational identification. In K. Murnigham (Ed) Social psychology in organizations: Advances in theory and research (p.244-268). Edgewood cliffs, NJ: Prentice Hall.

[19]Kuhn, T. (1962) The Structure of Scientific Revolutions. University of Chicago Press

[20]Leva M.C., McDonald N., Corrigan S., and Ulfvengren P. (2011) The challenge of system change in aviation: The MASCA project. Proceedings of ESREL 2011 edited by C. Berenguer and C.G. Soares published by Taylor and Francis Group.

[21] Maurer, R. (1996). Beyond the wall of resistance: Unconventional strategies that build support for change. Austin, TX: Bard Books, Inc.

[22] Mayer, R.C., Davis, J.H., and Schoorman, F.D. (1995) An integrative model of organizational trust. Academy of Management Review 20, 709-734 cited in Conchie, S.M., Taylor, P.J. \& Charlton, A. (2011) Trust and distrust in safety leadership: Mirror reflections? Safety Science 49, 1208 - 1214

[23] McDonald, N. (2006) Organizational Resilience and Industrial Risk in, editor(s) Erik Hollinagel David D Woods Nancy Leverson, Resilience Engineering, Concepts and Precepts, Surrey UK, Adgate Publishing,pp155 - 180.

[24] McDonald, N. (2009) Transformation: Human Factors and Organization Theory. HILAS Book outline. HILAS project, EC FP7, Contract No: 516181. Brussels: European Commission.

[25] McDonald, N. (2009) Strategic issues in the design, operation and regulation of large integrated operational systems. APRG, Trinity College Dublin Internal Paper.

[26] Mimosa (1998-2011). OSA CBM standard. www.mimosa.org

[27] Morrison, R., Grommes, P. and McDonald, N. (2007) Knowledge Space model - Human factors and operability concepts in the system of aviation industry, CEAS conference, Berlin, Germany.

[28] Nonaka, I., Toyoma, R. and Konno, N. (2002) The SECI, Ba and leadership: a unified model of dynamic knowledge creation. In S. Little, P. Quintas and T. Ray (Eds) Managing knowledge: an essential reader. London: Sage.

[29] Nonaka, I. (1994). A dynamic theory of organizational knowledge creation. Organization Science, 5(1), 14-37.

[30]Pascale, R., Millemann, M., and Gioja, L. (1997). Changing the way we change. Harvard Business Review, Vol. 75, No. 6, November/December, pps. 127-139.

[31]Pettigrew, A. (1985). The Awakening Giant: Continuity and Change at ICI. Oxford: Basil Blackwell.

[32] Pettigrew, A. and Whipp R. (1991). Managing change for competitive success. Oxford: Basil Blackwell.

[33] Porras, J. I. and Robertson, P. J. (1983). Organization development: theory, practice, and research. In M. D. Dunnette \& L. M. Hough (Eds.). The handbook of industrial and organizational psychology, 3, (pp. 719-822). Palo Alto, CA: Consulting Psychologists Press.

[34]Porter, M. (2008) On Competition, Updated and Expanded Edition. Harvard Business School Press

[35] Poortinga, W. and Pidgeon, N.F. (2003) Exploring the Dimensionality of Trust in Risk Regulation. Risk Analysis 23 (5), 961-972

[36] Seely Brown, J. and Duguid, P. (2001) Knowledge and Organization: A Social-Practice Perspective. Organization Science March/April 12 (2) 198-213

[37] Senge, P. (1990) The Fifth Discipline. The Art and Practice of the Learning Organization. London: Random House 
[38] Sitkin, S.B. and Stickel, D. (1996). The Road to Hell: The Dynamics of Distrust in an Era of Quality. In R.M. Kramer \& T.R. Tyler (Eds) Trust in Organizations, Frontiers of Theory and Research. California: Sage.

[39] Strebel, P (1996) Why do employees resist change? Harvard Business Review 74, 86-92

[40] Tan, H. and Chee, D (2005) Understanding interpersonal trust in Confucian infleuenced society: an exploratory study. International Journal of Cross Cultural Management 5, 197212

[41]Tatem D6102: Morrison R., McDonald N., Grommes P., Baranzini D., Liston P., and Ward M. (2006): Specification of the Knowledge Space Model. European Commission Framework programme. Contract No: AIP3-CT-2004-502909: Brussels: European Commission.

[41] Tatem D6103: Morrison, R, Grommes, P. and McDonald, D. (2008) Implementing the Knowledge Space Model. European Commission Framework programme. Contract No: AIP3-CT2004-502909: Brussels: European Commission.

[42] Tatem User Road Show (2005) Technologies and Techniques for New Maintenance Concepts. European Commission Framework programme. Contract No: AIP3-CT-2004-502909: Brussels: European Commission.

[43] Törner, M. (2011) The 'social-physiology' of safety. An integrative approach to understanding organizational psychological mechanisms behind safety performance. Safety Science 49, 1262-1269

[44] Ward, M. (2005) Contributions to Human Factors from three case studies in aircraft maintenance. Doctoral Thesis, Trinity College Dublin

[45] Ward, M., McDonald, N., Morrison, R., Gaynor, D., and Nugent, T. (2010) A Performance improvement case study in aircraft maintenance and its implications for hazard identification. Ergonomics, Special Edition: Human Factors in Aviation. Vol. 53, Issue 2, Pages $247-267$.

[46] Wenger, E. (1998) Communities of practice. Learning, meaning, and identity. Cambridge University Press.

[47]Zacharatos, A., Barling, J., and Iverson, R.D., (2005) High performance work systems and occupational safety. Journal of Applied Psychology 90, 77-93

[48]Zucker, L.G. (1986) Production of trust: Institutional sources of economic structure, 1840-1920. In B.M. Staw \& L.L. Cummings (Eds) Research in organizational behaviour (Vol. 8 p.53-111). Greenwich, CT: JAI. 